\title{
Update on Chemotherapy in the Treatment of Urothelial Carcinoma
}

\author{
Carrie Costantini and Frederick Millard \\ Moores UCSD Cancer Center, University of California, San Diego, 3855 Health Sciences Drive Mail Code 0987, \\ San Diego, CA 92093-0987, USA
}

Received 6 June 2011; Accepted 31 August 2011

Academic Editor: Karim Kader

\begin{abstract}
Urothelial carcinoma is the fifth most common malignancy diagnosed each year in the United States. Neoadjuvant and adjuvant chemotherapy are given to decrease the risk of recurrent or metastatic disease with the more robust clinical data supporting the former. Bladder preservation utilizes a trimodality approach with maximal transurethral resection followed by concurrent chemotherapy and radiation and is appropriate for select patients. Gemcitabine and cisplatin is the current standard of care for first-line treatment in fit patients with metastatic disease. Optimal second-line therapy remains undefined, and targeted agents are under investigation. Clinical trial participation should be encouraged in patients with urothelial carcinoma of the bladder to help improve treatment regimens and outcomes. Synopsis. Chemotherapy is commonly used in the treatment of urothelial carcinoma of the bladder. This paper will review the role of chemotherapy in the neoadjuvant, adjuvant, bladder sparing, and metastatic settings.
\end{abstract}

KEYWORDS: chemotherapy, urothelial carcinoma, bladder cancer 


\section{INTRODUCTION}

Urothelial carcinoma is the fifth most common malignancy diagnosed in the United States with an estimated 70,530 new cases and 14,680 deaths per year [1]. Approximately 30\% of patients present with muscle invasive urothelial carcinoma of the bladder (UCB) [2] and 5\% have metastatic disease at presentation. Radical cystectomy is the usual treatment of choice for muscle-invasive bladder cancer in the United States, although bladder sparing approaches have gained ground in recent years. Despite adequate local control, the overall survival (OS) after local therapy for muscle invasive UCB is suboptimal with five-year OS rates for pathologic T2 disease of 52-77\%, T3 disease 40-64\%, and T4 or node-positive disease 26-44\% [3]. Most patients who succumb to bladder cancer ultimately die due to distant disease. Thus, micrometastatic disease at time of local therapy is a major challenge. The role of systemic therapy is reviewed here in four contexts: neoadjuvant, adjuvant, bladder sparing with radiation, and metastatic disease.

\section{NEOADJUVANT CHEMOTHERAPY}

Integration of chemotherapy with surgery has been a dominant paradigm in oncology for decades, improving cure rates in common epithelial neoplasms such as breast and colon cancer as well as rarer mesenchymal tumors such as osteosarcoma and Ewing sarcoma. Progress in genitourinary cancers has been slower. Neoadjuvant (presurgical) chemotherapy offers potential advantages over adjuvant therapy, for example, early treatment of systemic micrometastases, potential downstaging of the primary and regional disease, and an in vivo assessment of chemosensitivity. The neoadjuvant approach also avoids potential delay in systemic treatment due to postoperative complications. This is a particular problem in bladder cancer, where as many as 58\% of patients may have postsurgical complications after radical cystectomy [15], potentially preventing timely administration of chemotherapy.

Several studies have evaluated the role of neoadjuvant chemotherapy in muscle-invasive UCB. The two most influential trials are discussed first. In November 1989, the Medical Research Council Advanced Bladder Cancer Working Party and the Genitourinary Group of the European Organisation for Research and Treatment of Cancer (EORTC) initiated an international study which randomized 976 patients with clinical T2 grade 3, T3-4a, N0-Nx tumors who were undergoing either cystectomy or definitive radiation therapy to receive 3 cycles of neoadjuvant cisplatin, methotrexate, and vinblastine (CMV) or no chemotherapy. Although originally reported as a negative trial [16], with longer followup a statistically significant survival advantage emerged [17]. There was a 5.5\% absolute difference in 3-year survival, and median OS for the chemotherapy group was 44 months versus 37.5 months in the control group. Pathological complete responses (CR) were significantly higher $(32.5 \%$ versus $12.3 \%)$ in the neoadjuvant chemotherapy group. They reported a chemotherapy mortality of $1 \%$ while operative mortality was equivalent in the two groups. The interpretation of these data are limited somewhat by the inclusion of both cystectomy and radiationtreated patients.

The US Intergroup Trial (SWOG 8710) randomized 307 patients with T2-T4a UCB to surgery alone or 3 cycles of neoadjuvant methotrexate, vinblastine, doxorubicin, and cisplatin (MVAC) [18]. With a median followup of 8.7 years, risk of death was $33 \%$ greater in those treated with surgery alone (hazard ratio 1.33). The median survival was 77 months in the chemotherapy group compared to 46 months with surgery alone. Thirty-eight percent of patients treated with preoperative MVAC had a pathological CR at surgery versus $15 \%$ in the surgery-alone group. Of those patients with a pT0 at surgery, $85 \%$ were alive at 5 years. Of note, this trial took 11 years to fully accrue, no doubt a record and indicative of the greatest hurdle to progress in bladder cancer.

The Nordic Urothelial Cancer Group combined the results of two trials to evaluate cisplatin-based combination neoadjuvant chemotherapy on 620 patients with T1 grade 3, T2-T4aNx bladder tumors [19]. The chemotherapy was cisplatin with doxorubicin in one study and cisplatin and methotrexate in the other. With a median followup of 4.7 years, an $8 \%$ absolute risk reduction in death at 5 years was observed, with an OS hazard ratio of 0.80 in favor of neoadjuvant therapy. 
Two meta-analyses of randomized trials of neoadjuvant chemotherapy for muscle-invasive bladder cancer have been published. The Advanced Bladder Cancer Meta-Analysis Collaboration used individual data from 3005 patients in 11 trials and reported a 5\% absolute survival improvement at 5 years with platinum-based combination neoadjuvant chemotherapy [20]. All but 196 patients received cisplatin [21]. They also demonstrated a 9\% improvement in 5-year disease-free survival (DFS). A similar outcome was seen in another meta-analysis with an absolute OS benefit of $6.5 \%$ (from 50 to 56.5\%) at 5 years [22]. Mortality attributed to neoadjuvant chemotherapy in this analysis was $1.1 \%$.

Based on the data from the metastatic setting where gemcitabine and cisplatin (GC) has replaced MVAC as preferred treatment due to similar response rates and reduced toxicity, GC has been studied in the neoadjuvant setting in small, nonrandomized trials. Herchenhorn et al. reported a single institution study with 3 cycles of neoadjuvant GC in 22 patients with T2-T4 disease and found a combined partial and complete radiographic response in $70 \%$ of patients [23]. Pathologic CR was found in 26.7\% (4/15) of the patients who went onto surgery. Treatment was well tolerated with no deaths attributed to chemotherapy. With 26 month followup, the estimated median OS was 36 months. In a retrospective review from the Memorial Sloan-Kettering Cancer Center, 42 patients were treated with 4 cycles of neoadjuvant GC, with downstaging to pT0 in $26 \%$ of patients and to no residual muscle-invasive disease $(<\mathrm{pT} 2)$ in $36 \%$ [24]. All the patients achieving $<$ pT2 remained disease-free at the median followup of 30 months. However, despite widespread adoption, neoadjuvant $\mathrm{GC}$ has not been validated in prospective, randomized studies.

Overall, the data indicate that neoadjuvant cisplatin-based chemotherapy is feasible and provides a modest survival benefit. Therefore, it is appropriate to consider neoadjuvant chemotherapy as a standard of care in muscle-invasive bladder cancer planned for surgical intervention.

\section{ADJUVANT CHEMOTHERAPY}

Postoperative adjuvant chemotherapy is another systemic treatment option for patients with muscle-invasive bladder cancer. Immediate surgical intervention provides debulking and relief of local symptoms. Another advantage is the availability of pathologic staging to more accurately assess risk, as clinical staging is often inaccurate. A large case series of 3393 patients with UCB treated with radical cystectomy reported clinical understaging in $50 \%$ of patients and pathologic downstaging in 18\% [25].

Several trials have examined the role of adjuvant chemotherapy in muscle-invasive disease with conflicting results. A trial with single-agent cisplatin failed to show a survival advantage [26]. Skinner et al. reported 91 patients with pT3-4 or node-positive UCB randomized to adjuvant chemotherapy (predominantly cisplatin, doxorubicin, and cyclophosphamide) versus cystectomy alone [27]. They found a significant improvement in time to progression with chemotherapy but no difference in 5-year OS. However, 25\% of patients randomized to adjuvant chemotherapy never received treatment. Another limitation of this study was the variability of chemotherapeutic regimens.

Adjuvant MVAC or MVEC (with epirubicin instead of doxorubicin) was studied in 49 patients with pT3-4a or positive lymph nodes using 3 cycles of chemotherapy after cystectomy and demonstrated improved DFS at an interim analysis, resulting in the trial being stopped early [28]. It is important to note that only 18 of the 26 patients randomized to the chemotherapy group received adjuvant treatment. The results were updated several years later with an additional 117 nonrandomized patients and continued to show a progression-free survival (PFS) advantage for those who received adjuvant chemotherapy [29]. This group also published long-term survival data on the initial 49 patients with a median followup of 160 months [30]. In an intent-to-treat analysis, the median PFS was 66.9 versus 11.6 months (hazard ratio 2.84, $P=0.002$ favoring adjuvant chemotherapy) and tumor-specific survival of 71.8 versus 20.4 months with a hazard ratio of $2.52(P=0.007)$ favoring adjuvant treatment. Five-year OS was $38.5 \%$ in the treatment arm versus $17.4 \%$ in the control arm. Forty-four percent of patients in the control arm did receive combination chemotherapy at progression. 
Adjuvant cisplatin and methotrexate (CM) was compared to MVEC in a phase III trial with 327 patients with pT3a-4a tumor or node-positive UCB [31]. Similar 5-year PFS, tumor-specific survival, and OS rates were reported, but with significantly reduced hematologic toxicity in the CM arm.

Trials such as these are often criticized for small sample sizes and lack of statistical power. Another common criticism is that several studies were closed early after interim analyses. Two meta-analyses on adjuvant chemotherapy for muscle invasive bladder cancer have been reported. The Advanced Bladder Cancer Meta-Analysis Collaboration obtained individual patient data from 491 patients in 6 randomized trials of adjuvant cisplatin-based chemotherapy and found an overall hazard ratio for survival of $0.75(P=$ $0.019)$ favoring adjuvant therapy [32]. This corresponds to an absolute improvement in survival of $9 \%$ at 3 years. However, the lack of robustness of the data prevented the authors from making a formal endorsement of adjuvant treatment. Another meta-analysis of 5 trials found a similar improvement in OS with adjuvant chemotherapy with a hazard ratio of $0.74(P=0.001)$ [33]. Svatek et al. reported retrospective data from 11 centers with 3947 patients with high-risk UCB treated with cystectomy, of which $23.6 \%$ received adjuvant chemotherapy [34]. Adjuvant chemotherapy was associated with improved survival (hazard ratio $0.83, P=$ 0.017) with increasing benefit seen with higher-risk subgroups.

Several other large randomized trials have been reported in abstract form. An Italian group randomized 194 patients with pT2 grade 3, pT3-4, N0-2 UCB to adjuvant GC versus chemotherapy at relapse and did not show a significant difference in DFS and OS [35]. However, the trial closed early due to poor accrual, enrolling only $32 \%$ of the target sample size. A US study randomized 114 patients with pT1-2, N0, p53 positive tumors to 3 cycles of adjuvant MVAC or observation [36]. Further accrual was halted after a planned interim futility analysis showed no significant differences in recurrence-free survival. Only $67 \%$ of patients randomized to MVAC received all 3 cycles, and $12 \%$ received none, further limiting the power of the study. The Spanish Oncology Genitourinary Group (SOGUG) 99/01 study also closed prematurely due to poor recruitment after 7 years and failure to meet goal enrollment of 340 patients. They randomized 142 patients with pT3-4 or node-positive disease to 4 cycles of paclitaxel, gemcitabine, and cisplatin (PGC) versus observation. With median follow up of 51 months, a 5 year OS benefit with chemotherapy of $60 \%$ versus $31 \%(P<0.0009)$ was reported [37].

The EORTC 30994 phase III trial comparing immediate versus deferred chemotherapy (MVAC, high-dose MVAC every 14 days with growth factor support, or GC) after radical cystectomy in patients with pT3-4 or node-positive UCB planned to enroll 660 patients but closed after 7 years with only 284 accrued [38]. No results have been reported as of yet. Similarly, Cancer and Leukemia Group B (CALGB) 90104 intended to compare sequential doxorubicin and gemcitabine followed by paclitaxel and cisplatin with GC alone in the adjuvant setting, but closed due to slow accrual after enrolling only 42 subjects.

While data from meta-analyses suggest a possible benefit to adjuvant chemotherapy in muscle invasive UCB, further data are needed before firm recommendations can be made. Available clinical trial data are underpowered and more recent attempts at large randomized trials have accrued poorly. Possible contributors to this phenomenon include the frequent occurrence of postoperative complications preventing adjuvant treatment, the frequent coexistence of chronic kidney disease in this population limiting the use of cisplatin, and lack of interest on the part of urologists, medical oncologists, and/or patients. In the absence of robust data, most GU oncologists offer adjuvant therapy to fit patients when neoadjuvant therapy is not an option. Whether this should also be done for patients not suitable for cisplatin is an even murkier question. Ongoing clinical trial participation is essential, but the field lacks momentum at this point in time.

\section{CHEMOTHERAPY IN BLADDER PRESERVATION}

Bladder preservation may be accomplished in appropriately selected patients with muscle-invasive UCB without compromising outcomes using a trimodality approach with maximal transurethral resection (TUR) followed by concurrent chemotherapy and radiation therapy. Optimal application of this approach requires close coordination among the urologist, medical oncologist, and radiation oncologist. Appropriate candidates have T2-4a and clinically node-negative disease, primary tumors amenable to complete or near 
complete TUR, no hydronephrosis and adequate renal function to tolerate cisplatin. Salvage cystectomy is required for persistent or recurrent disease at repeat cystoscopy, either during or after chemoradiotherapy (CRT), and is typically necessary in approximately one-third of patients [39]. The largest single-institution series from the University of Erlangen (415 patients) showed that early tumor stage and a complete TUR are the most important factors predicting CR and survival [40]. Chemotherapy alone is not an appropriate treatment for muscle-invasive UCB being treated for cure.

Two randomized trials have shown that concurrent CRT is more effective than radiation alone in reducing local recurrences, but an improvement in OS has not yet been demonstrated [41, 42]. There are no prospective randomized trials of CRT versus cystectomy. Thus, knowledge of this approach is derived primarily from case series and phase I/II trials.

The largest US series comes from the Massachusetts General Hospital. They have reported on 190 patients using concurrent cisplatin-containing chemotherapy and radiotherapy after TUR [43]. Surgical candidates with less than a cT0 response to CRT or those unable to tolerate CRT went to salvage cystectomy. With a median followup of 6.7 years, the 5- and 10-year disease-specific survival rate was 63\% and 59\% with the 5- and 10-year disease-specific survival rate for patients with an intact bladder of $46 \%$ and $45 \%$, respectively. The pelvic failure rate was $8.4 \%$. One-third of patients treated on protocol with the goal of bladder sparing ultimately required a cystectomy.

The concept of induction chemotherapy in bladder preservation has been explored by the Radiation Therapy Oncology Group (RTOG) in serial phase II studies. RTOG 89-03 randomized 123 patients with T2-4a, Nx UCB to 2 cycles of neoadjuvant cisplatin, methotrexate, and vinblastine (CMV) followed by concurrent CRT with cisplatin versus concurrent CRT with cisplatin alone; those with less than CR at cystoscopy underwent cystectomy and those with a CR underwent additional CRT [44]. Seventy-four percent completed the treatment, and there was no significant difference in 5-year OS, OS with a functioning bladder, freedom from metastatic disease, freedom from pelvic recurrence, or improvement in clinical CR rate. Thus, the role of induction chemotherapy has not been established.

Other chemotherapeutic agents have been evaluated with and without cisplatin to enhance radiosensitivity. Zietman et al. described 18 patients with T2-4a UCB treated with twice daily radiation with cisplatin and 5-fluorouracil (5-FU) [45]. Of note, 15 of the patients were also treated with adjuvant CMV. A complete response to CRT was seen in 14 of 18 patients, the 3-year OS was 83\%, and the 3year survival with a native bladder was $78 \%$. One hundred twelve patients with muscle-invading or highrisk T1 UCB were enrolled in a study of concurrent cisplatin and 5-FU with radiotherapy that reported 5 year overall and cause-specific survival for all patients of $74 \%$ and $82 \%$, respectively, [46]. Eighty-eight percent of patients had a CR, and, of all surviving patients, 82\% maintained their own bladder. RTOG 95-06 evaluated 34 patients with clinical stage T2-4a, Nx UCB treated with twice daily radiation with cisplatin and 5-FU with an induction course followed by consolidation if a CR was achieved [47]. With a median followup of 29 months, $67 \%$ of patients had a CR after induction treatment, and the actuarial 3-year OS was $83 \%$ with $66 \%$ of patients surviving with an intact bladder. However, $21 \%$ of patients developed grade $3-4$ hematologic toxicity during therapy.

RTOG 99-06 evaluated 80 patients with T2-4a UCB treated with twice daily radiation with paclitaxel and cisplatin (induction CRT) [48]. If repeat biopsy showed $<\mathrm{T} 1$ disease, they were treated with consolidation chemoradiotherapy; if $>\mathrm{T} 1$, then cystectomy was advised. All patients received adjuvant GC. Ninetyfive percent completed the induction treatment with $26 \%$ grade 3-4 toxicities. The postinduction CRT complete response rate was $81 \%$. Adjuvant GC was completed in $70 \%$ of patients with grade 3 toxicities in $46 \%$, grade 4 in $26 \%$, and 1 death due to thrombotic thrombocytopenia purpura and hemorrhagic stroke. With a median followup of 49.4 months, the 5-year OS rate was 56\%, and disease-specific survival rate was $71 \%$.

RTOG 97-06 also looked at twice daily radiation but added adjuvant CMV chemotherapy [49]. They described 47 patients with T2-4a, N0 UCB after TUR treated with induction CRT with twice daily radiation with cisplatin. If there was no evidence of disease at repeat cystoscopy, they were treated with consolidation CRT. Patients with residual tumor went onto cystectomy. After either consolidation CRT or cystectomy, patients were treated with 3 cycles of CMV chemotherapy. The CR rate after induction therapy was $74 \%$. 
The projected 3-year OS and bladder-intact survival was $61 \%$ and $48 \%$, respectively. However, only $45 \%$ of patients completed three cycles of adjuvant chemotherapy.

Gemcitabine as a radiosensitizer has been shown to be well tolerated and feasible in UCB in earlyphase trials $[50,51]$. A Phase II trial of concurrent weekly gemcitabine with radiation was reported in 50 patients with T2-3 UCB with $92 \%$ of patients tolerating 4 doses of gemcitabine and $88 \%$ achieving a CR [52]. At a median followup of 36 months, the 3-year cancer-specific survival was $82 \%$ with OS of $75 \%$. Eighty-nine percent of survivors had intact bladders. There was no difference in late toxicity scores at 2 years as compared to baseline.

In appropriately selected patients, bladder preservation with TUR, chemotherapy, and radiation are feasible and produces high rates of CR with acceptable disease control and OS with intact bladders. Concurrent cisplatin-based radiation has the most supporting data, but newer agents are being evaluated. The value of induction chemotherapy has not been established, and the value of postradiation adjuvant therapy, while conceptually attractive, lacks sufficient data for definitive conclusions. Careful, continued surveillance with cystoscopy both during and after CRT is mandatory.

\section{METASTATIC DISEASE}

UCB is considered a chemotherapy-sensitive disease with response rates in fit patients approaching $50 \%$. Systemic chemotherapy remains the standard of care for patients with metastatic UCB. The principle challenge in this context is the high frequency of impaired performance status, renal dysfunction, and other comorbidities.

Historically, the standard first-line therapy for fit patients in the combination chemotherapy era has been MVAC. MVAC has been compared to both cisplatin alone and a combination of cisplatin, cyclophosphamide, and doxorubicin (CISCA) with superior response rates and OS [53, 54], with median survival of 12.5 months [53]. However, MVAC is associated with significant toxicity including myelosuppression, neutropenic fever and sepsis, mucositis, nausea, vomiting, and a 3-4\% toxic death rate [53, 55]. These side effects and the complex schedule often limit its use. However, $3.7 \%$ of patients randomized to MVAC in one trial were alive and disease-free at 6 years, indicating it may be curative in a small fraction of patients [56]. EORTC 30924 studied standard MVAC versus dose-intensified MVAC with growth factor support (HD-MVAC) in an attempt to improve outcomes as well as decrease toxicity. A total of 263 patients were randomized and, while there was no statistically significant difference in response rates or OS, there was borderline improved PFS (9.1 versus 8.2 months) as well as less leukopenia, neutropenic fever, and mucositis with HD-MVAC [55].

Due to significant toxicities with MVAC, other regimens have been investigated in the first-line metastatic setting. A phase III trial of 220 patients demonstrated that MVAC was more effective than docetaxel and cisplatin with improved response rates, time to progression, and median survival [57]. After encouraging phase II data [58-60], gemcitabine and cisplatin (GC) were compared to MVAC in a randomized phase III trial with 405 patients [61]. While the study was not powered to show equivalency, overall response rate, time to progression, and median survival were similar for both regimens, with less grade 3-4 neutropenia, neutropenic fever, sepsis, mucositis and alopecia in the GC arm. The toxic death rate was 3\% with MVAC and 1\% with GC. Long-term followup after 5 years demonstrated continued similar survival between the two arms [62]. Based on these results, GC is now considered the standard-of-care first-line therapy for fit patients with metastatic bladder cancer.

Attempts to improve upon this regimen have included adding a third drug to GC. The SOGUG found the combination of paclitaxel, gemcitabine, and cisplatin (PGC) to be feasible with an overall response rate of $77.6 \%$ (with $28 \%$ CRs) in 61 patients [63]. To more fully evaluate this tactic, the EORTC 30987/Intergroup Study randomized 627 patients to either PGC or GC with a primary endpoint of OS [64]. The first report in abstract form demonstrated an improved response rate with PGC (57\% versus 46\%) but only a trend in favor of OS (15.7 versus 12.8 month, $P=0.10)$. Of note, the study was powered to detect 
a 4-month difference in survival. There was more thrombocytopenia on the GC arm, while PGC was associated with increased leukopenia, neutropenia, and febrile neutropenia.

The role of carboplatin has been evaluated in both cisplatin eligible and ineligible populations. Several studies have compared carboplatin- and cisplatin-containing regimens in patients with adequate renal function. A phase II trial in 55 patients comparing MVEC versus MVECa (methotrexate, vinblastine, epirubicin, and carboplatin) demonstrated that MVEC had higher response rates compared to the carboplatin-containing regimen [65]. Bellmunt et al. reported 47 patients randomized to MVAC or MCAVI (methotrexate, carboplatin, vinblastine) and showed that MCAVI was less toxic but also less effective with median DFS favoring MVAC (16 months versus 9 months) [66]. An Eastern Cooperative Oncology Group (ECOG) study attempted to compare carboplatin and paclitaxel (CP) to MVAC, although median survival was similar, the study closed prematurely due to slow accrual [67], and no definite conclusions can be made. GC was compared to gemcitabine and carboplatin in a phase II study in 110 patients with the primary objective to compare toxicities [68]. Toxicities were similar, and median survival was not significantly different at 12.8 months in the GC arm versus 9.8 months in the gemcitabine/carboplatin arm, although the study was inadequately powered for this endpoint. Based on this evidence, cisplatin remains the standard of care in patients with adequate renal function.

In patients unfit for cisplatin, carboplatin-based regimens have demonstrated activity [69-73]. However, there is significant variability in the definition of patients "unfit" for cisplatin. A recent abstract reviewed 14 publications regarding cisplatin ineligibility and noted wide variation in terms of renal function, performance status, age, and comorbidities [74]. The authors proposed a consensus definition of cisplatin ineligibility criteria for clinical trials including (1) ECOG performance status of 2, (2) creatinine clearance $<60 \mathrm{~mL} / \mathrm{min}$, (3) Common Terminology Criteria for Adverse Events (CTCAE) Grade $\geq 2$ hearing loss, (4) CTCAE Grade $\geq 2$ neuropathy. Also confounding the problem is the fact that current formulas estimating creatinine clearance tend to underestimate the measured creatinine clearance, especially in those patients older than 65 years [75].

First-line nonplatinum combinations have been evaluated in phase II trials and have shown activity with response rates of $54-70 \%$ with gemcitabine and paclitaxel [76, 77]. In one series of 36 patients, $14 \%$ developed grade 3-5 pulmonary toxicity [77]; however this degree of pulmonary toxicity was not seen in other trials $[76,78,79]$ and has not been an issue with this regimen in lung cancer patients [80]. A phase II trial of 46 patients treated with pemetrexed (a multitargeted antifolate agent) and gemcitabine demonstrated a response rate of $32 \%$ and a median OS of 12.4 months [81]. However, this was at the expense of $75 \%$ grade 3-4 myelosuppression. There are no reported phase III comparisons of nonplatinum regimens.

Several single agents have been evaluated in the second-line setting in phase II trials (Table 1). As one might expect, the problem of "unfit" patients is even more challenging in this context. Single agents including ifosfamide [4], docetaxel [5], and gemcitabine [6, 7] have been evaluated in small studies with modest results. Second-line weekly paclitaxel has a low response rate $[8,9]$, though one study reported 38\% with stable disease [9]. Pemetrexed has been reported to have a response rate of 28\%, a median OS of 9.6 months and was well tolerated in a phase II trial of 47 pretreated patients [10]. However, another study of 13 patients only showed a $8 \%$ response rate [11]. Single-agent ixabepilone also has modest activity [12]. An encouraging phase II trial of second-line nab-paclitaxel was recently presented, reporting a response rate of $33 \%$ with a $58 \%$ clinical benefit rate [13]; this is deserving of further investigation.

Vinflunine is a novel microtubule inhibitor that has been studied in a phase III trial in the second-line setting for metastatic UCB, where 370 patients were randomized to vinflunine plus best supportive care versus best supportive care alone [14]. In the eligible population of 357 patients, the median OS was significantly longer for the vinflunine group (6.9 versus 4.3 months). Grade 3-4 toxicities included neutropenia (50\%), febrile neutropenia (6\%), anemia (19\%), fatigue (19\%), and constipation (16\%). There was one drug-related death on study. Vinflunine is approved for use in Europe but not in the United States.

Second-line combination therapies have also been evaluated. Two phase II trials looked at various doses of gemcitabine and paclitaxel in platinum-pretreated patients, with response rates of $30 \%$ and $33 \%$ and median survival of 11.5 and 11.3 months, respectively [82, 83]. A phase III trial of 102 patients compared 
TABLE 1: Second-line single agent chemotherapy trials in advanced urothelial carcinoma.

\begin{tabular}{lcccc}
\hline Reference & Agent & Subjects & Response rate (\%) & Survival (months) \\
\hline Witte et al. [4] & Ifosfamide & 56 & 20 & 5.5 \\
McCaffrey et al. [5] & Docetaxel & 30 & 13 & 9 \\
Lorusso et al. [6] & Gemcitabine & 35 & 23 & 5 \\
Albers et al. [7] & Gemcitabine & 30 & 11 & 8.7 \\
Vaughn et al. [8] & Paclitaxel & 31 & 10 & 7.2 \\
Joly et al. [9] & Paclitaxel & 45 & 9 & 7 \\
Sweeney et al. [10] & Pemetrexed & 47 & 28 & 9.6 \\
Galsky et al. [11] & Pemetrexed & 13 & 8 & Not reported \\
Dreicer et al. [12] & Ixabepilone & 45 & 12 & 8 \\
Sridhar et al. [13] & Nab-paclitaxel & 48 & 33 & Not reported \\
Bellmunt et al. [14] & Vinflunine & 307 & 9 & 6.9 \\
\hline
\end{tabular}

"short term" treatment with weekly gemcitabine and paclitaxel in a 21-day cycle with a maximum of 6 cycles versus continuing the same treatment until disease progression [79]. There was no difference in response rates (37.5 versus $41.5 \%$ ) and OS (7.8 versus 8 months) in the two arms; there was more grade 3-4 anemia and two treatment-related deaths in the prolonged treatment arm. The combination of ifosfamide and docetaxel in 20 cisplatin-pretreated patients demonstrated an overall response rate of $25 \%$ including 4 CRs [84]. Given the lack of definitive randomized data, there remains no well-defined standard of care for second-line chemotherapy for metastatic bladder cancer.

\section{NOVEL AND TARGETED AGENTS}

Her2/neu is variably expressed on urothelial carcinomas and has been evaluated as a possible therapeutic target. One study screened 109 patients with advanced UCB and found that Her2-positive patients (52\%) had more metastatic sites and higher rates of visceral disease than Her2-negative patients [85]. Forty-four patients were treated with trastuzumab, paclitaxel, carboplatin, and gemcitabine with an overall response rate of $70 \%$ with $57 \%$ confirmed responses. Median survival was 14.1 months. Toxicities included $93 \%$ grade 3-4 myelosuppression, $14 \%$ grade 3 sensory neuropathy, $22.7 \%$ grade $1-3$ of cardiotoxicity (4.5\% grade 3 ), and 3 therapy-related deaths.

Table 2 summarizes the ongoing clinical trials with targeted agents in urothelial carcinoma. Epidermal growth factor receptor (EGFR) expression has been demonstrated on 50\% of bladder tumors [86], and trials are ongoing with cetuximab. Vascular endothelial growth factor (VEGF) receptors are another potential target. Trials are evaluating the use of bevacizumab both in the metastatic and neoadjuvant setting as well as other anti-VEGF agents. However, a recent phase II experience with sunitinib in advanced UCB was disappointing, with only 4 responses in 77 patients [87].

Given the experience in nonmuscle-invasive UCB with BCG and interferon, there has been interest in the application of immunotherapy to more advanced bladder cancer as well. Ongoing trials are evaluating a human chorionic gonadotropin- $\beta$ vaccine (Celldex Therapeutics, CDX1307-03, NCT01094496), and the anticytotoxic T-lymphocyte-associated antigen 4 antibody ipilimumab (NCT00462930), both in the neoadjuvant setting.

\section{CONCLUSIONS}

Chemotherapy continues to play an important role in the treatment of urothelial carcinoma in a variety of settings. Neoadjuvant cisplatin-based combination chemotherapy has demonstrated a small but significant 
TABLE 2: Ongoing clinical trials with targeted agents in urothelial carcinoma.

\begin{tabular}{|c|c|c|c|c|}
\hline Phase & Cohort & Subjects & Treatment & Protocol number \\
\hline $\mathrm{I} / \mathrm{II}$ & $\begin{array}{l}\text { Noncystectomy candidates } \\
\text { undergoing radiation in } \\
\text { muscle-invasive disease }\end{array}$ & 88 & Paclitaxel \pm trastuzumab & NCT00238420 \\
\hline I & First line metastatic/advanced & 25 & GC with lapatinib & NCT00623064 \\
\hline II/III & $\begin{array}{l}\text { Maintenance after first-line } \\
\text { metastatic/advanced }\end{array}$ & 204 & Lapatinib versus placebo & NCT00949455 \\
\hline II & First-line metastatic/advanced & 86 & $\mathrm{GC} \pm$ cetuximab & NCT00645593 \\
\hline II & First-line metastatic/advanced & 51 & GC with bevacizumab & NCT00588666 \\
\hline III & First-line metastatic/advanced & 500 & $\mathrm{GC} \pm$ bevacizumab & NCT00942331 \\
\hline II & Neoadjuvant/adjuvant & 25 & $\begin{array}{l}\text { Neoadjuvant GC with bevacizumab } \\
\text { Adjuvant paclitaxel with } \\
\text { bevacizumab }\end{array}$ & NCT00268450 \\
\hline II & Neoadjuvant & 45 & GC with sunitinib & NCT00847015 \\
\hline II & Adjuvant & 33 & $\begin{array}{l}\text { Sunitinib (after neoadjuvant } \\
\text { platinum-based chemotherapy) }\end{array}$ & NCT01042795 \\
\hline II & $\begin{array}{l}\text { First-line cisplatin ineligible } \\
\text { metastatic/advanced }\end{array}$ & 41 & Sunitinib & NCT01118039 \\
\hline II & First-line metastatic/advanced & 63 & GC with sunitinib & NCT01089088 \\
\hline II & Neoadjuvant & 45 & GC with sorafenib & NCT01222676 \\
\hline II & First line metastatic/advanced & 30 & GC with sorafenib & NCT00461851 \\
\hline I & $\begin{array}{l}\text { Non-cystectomy candidates } \\
\text { undergoing radiation in muscle } \\
\text { invasive disease }\end{array}$ & 18 & Sorafenib & NCT00544609 \\
\hline II & $\begin{array}{l}\text { Second-line cisplatin pretreated } \\
\text { metastatic/advanced }\end{array}$ & 41 & Pazopanib & NCT01031875 \\
\hline II & Second- or third-line metastatic & 32 & Pazopanib with weekly paclitaxel & NCT01108055 \\
\hline Pilot & Neoadjuvant & 25 & Dasatinib & NCT00706641 \\
\hline II & $\begin{array}{l}\text { First-line cisplatin ineligible } \\
\text { metastatic/advanced }\end{array}$ & 122 & $\begin{array}{l}\text { Carboplatin/Gemcitabine } \pm \\
\text { vandetanib }\end{array}$ & NCT01191892 \\
\hline
\end{tabular}

*Data from http://www.clinicaltrials.gov/ accessed on April 2011.

survival benefit. Adjuvant chemotherapy can also be considered, although the data are less compelling. A bladder-sparing approach using chemoradiotherapy is appropriate in carefully selected patients. Chemotherapy remains the mainstay of treatment of metastatic urothelial carcinoma. Gemcitabine plus cisplatin is the current standard of care for first-line treatment in fit patients. Data are less robust for patients unfit for cisplatin, but carboplatin is often used. There are no conclusive data to support a standard second-line regimen, and current practice relies on phase II trials. Various targeted agents are currently under investigation. Development of less toxic, more effective agents is critical, and clinical trial participation needs to be prioritized.

\section{REFERENCES}

[1] A. Jemal, R. Siegel, J. Xu, and E. Ward, “Cancer statistics, 2010,” CA Cancer Journal for Clinicians, vol. 60, no. 5, pp. 277-300, 2010. 
[2] J. E. Rosenberg, P. R. Carroll, and E. J. Small, "Update on chemotherapy for advanced bladder cancer," Journal of Urology, vol. 174, no. 1, pp. 14-20, 2005.

[3] J. P. Stein, G. Lieskovsky, R. Cote et al., "Radical cystectomy in the treatment of invasive bladder cancer: longterm results in 1,054 patients," Journal of Clinical Oncology, vol. 19, no. 3, pp. 666-675, 2001.

[4] R. S. Witte, P. Elson, B. Bono et al., "Eastern cooperative oncology group phase II trial of ifosfamide in the treatment of previously treated advanced urothelial carcinoma," Journal of Clinical Oncology, vol. 15, no. 2, pp. 589-593, 1997.

[5] J. A. McCaffrey, S. Hilton, M. Mazumdar et al., "Phase II trial of docetaxel in patients with advanced or metastatic transitional-cell carcinoma," Journal of Clinical Oncology, vol. 15, no. 5, pp. 1853-1857, 1997.

[6] V. Lorusso, C. F. Pollera, M. Antimi et al., "A phase II study of gemcitabine in patients with transitional cell carcinoma of the urinary tract previously treated with platinum. Italian Co-operative Group on Bladder Cancer," European Journal of Cancer, vol. 34, no. 8, pp. 1208-1212, 1998.

[7] P. Albers, R. Siener, M. Haertlein et al., "Gemcitabine monotherapy as second-line treatment in cisplatinrefractory transitional cell carcinoma-Prognostic factors for response and improvement of quality of life," Onkologie, vol. 25, no. 1, pp. 47-52, 2002.

[8] D. J. Vaughn, C. M. Broome, M. Hussain, J. C. Gutheil, and A. B. Markowitz, "Phase II trial of weekly paclitaxel in patients with previously treated advanced urothelial cancer," Journal of Clinical Oncology, vol. 20, no. 4, pp. 937-940, 2002.

[9] F. Joly, N. Houédé, S. Noal et al., "Do patients with advanced urothelial carcinoma benefit from weekly paclitaxel chemotherapy? A GETUG phase II study," Clinical Genitourinary Cancer, vol. 7, no. 2, pp. E28-E33, 2009.

[10] C. J. Sweeney, B. J. Roth, F. F. Kabbinavar et al., "Phase II study of pemetrexed for second-line treatment of transitional cell cancer of the urothelium," Journal of Clinical Oncology, vol. 24, no. 21, pp. 3451-3457, 2006.

[11] M. D. Galsky, S. Mironov, A. Iasonos, J. Scattergood, M. G. Boyle, and D. F. Bajorin, "Phase II trial of pemetrexed as second-line therapy in patients with metastatic urothelial carcinoma," Investigational New Drugs, vol. 25 , no. 3, pp. 265-270, 2007.

[12] R. Dreicer, S. Li, J. Manola, N. B. Haas, B. J. Roth, and G. Wilding, "Phase 2 trial of epothilone B analog BMS-247550 (ixabepilone) in advanced carcinoma of the urothelium (E3800): a trial of the eastern cooperative oncology group," Cancer, vol. 110, no. 4, pp. 759-763, 2007.

[13] S. Sridhar, C. Canil, and S. Mukherjee, "Results of a phase II study of single agent nab-paclitaxel in platinumrefractory second line metastatic urothelial carcinoma (UC)," Journal of Clinical Oncology, vol. 29, supplement 7, abstract 241, 2011.

[14] J. Bellmunt, C. Théodore, T. Demkov et al., "Phase III trial of vinflunine plus best supportive care compared with best supportive care alone after a platinum-containing regimen in patients with advanced transitional cell carcinoma of the urothelial tract," Journal of Clinical Oncology, vol. 27, no. 27, pp. 4454-4461, 2009.

[15] N. Lawrentschuk, R. Colombo, O. W. Hakenberg et al., "Prevention and management of complications following radical cystectomy for bladder cancer," European Urology, vol. 57, no. 6, pp. 983-1001, 2010.

[16] "Neoadjuvant cisplatin, methotrexate, and vinblastine chemotherapy for muscle-invasive bladder cancer: a randomised controlled trial. International collaboration of trialists," The Lancet, vol. 354, pp. 533-540, 1999.

[17] R. R. Hall and Intl Collaboration of Trialists of the MRC Advanced Bladder Cancer Group, "Updated results of a randomized controlled trial of neoadjuvant cisplatin (C), methotrexate (M) and vinblastine (V) chemotherapy for muscle-invasive bladder cancer,' in Proceedings of the American Society of Clinical Oncology (ASCO '02), vol. 21, May 2002.

[18] H. B. Grossman, R. B. Natale, C. M. Tangen et al., "Neoadjuvant chemotherapy plus cystectomy compared with cystectomy alone for locally advanced bladder cancer," New England Journal of Medicine, vol. 349, no. 9, pp. 859-866, 2003.

[19] A. Sherif, L. Holmberg, E. Rintala et al., "Neoadjuvant cisplatinum based combination chemotherapy in patients with invasive bladder cancer: a combined analysis of two nordic studies," European Urology, vol. 45, no. 3, pp. 297-303, 2004.

[20] "Neoadjuvant chemotherapy in invasive bladder cancer: update of a systematic review and meta-analysis of individual patient data advanced bladder cancer (ABC) meta-analysis collaboration," European Urology, vol. 48, pp. 202-205, 2005.

[21] Advanced Bladder Cancer Meta-Analysis, "Neoadjuvant chemotherapy for invasive bladder cancer," in Cochrane Database of Systematic Reviews, John Wiley \& Sons, Chichester, UK, 2004. 
[22] E. Winquist, T. S. Kirchner, R. Segal, J. Chin, and H. Lukka, "Neoadjuvant chemotherapy for transitional cell carcinoma of the bladder: a systematic review and meta-analysis," Journal of Urology, vol. 171, no. 2 I, pp. 561-569, 2004.

[23] D. Herchenhorn, R. Dienstmann, F. A. Peixoto et al., "Phase II trial of neoadjuvant gemcitabine and cisplatin in patients with resectable bladder carcinoma," International Brazilian Journal of Urology, vol. 33, no. 5, pp. 630-638, 2007.

[24] A. Dash, J. A. Pettus, H. W. Herr et al., "A role for neoadjuvant gemcitabine plus cisplatin in muscle-invasive urothelial carcinoma of the bladder: a retrospective experience," Cancer, vol. 113, no. 9, pp. 2471-2477, 2008.

[25] R. S. Svatek, S. F. Shariat, G. Novara et al., "Discrepancy between clinical and pathological stage: external validation of the impact on prognosis in an international radical cystectomy cohort," British Journal of Urology International, vol. 107, no. 6, pp. 898-904, 2011.

[26] U. E. Studer, M. Bacchi, C. Biedermann et al., "Adjuvant cisplatin chemotherapy following cystectomy for bladder cancer: results of a prospective randomized trial," Journal of Urology, vol. 152, no. 1, pp. 81-84, 1994.

[27] D. G. Skinner, J. R. Daniels, C. A. Russell et al., "The role of adjuvant chemotherapy following cystectomy for invasive bladder cancer: a prospective comparative trial," Journal of Urology, vol. 145, no. 3, pp. 459-467, 1991.

[28] M. Stockle, W. Meyenburg, S. Wellek et al., "Advanced bladder cancer (stages pT3b, pT4a, pN1 and pN2): improved survival after radical cystectomy and 3 adjuvant cycles of chemotherapy. Results of a controlled prospective study," Journal of Urology, vol. 148, no. 2 I, pp. 302-307, 1992.

[29] M. Stöckle, S. Wellek, W. Meyenburg et al., "Radical cystectomy with or without adjuvant polychemotherapy for non- organ-confined transitional cell carcinoma of the urinary bladder: prognostic impact of lymph node involvement," Urology, vol. 48, no. 6, pp. 868-875, 1996.

[30] J. Lehmann, L. Franzaring, J. Thüroff, S. Wellek, and M. Stöckle, "Complete long-term survival data from a trial of adjuvant chemotherapy vs control after radical cystectomy for locally advanced bladder cancer," British Journal of Urology International, vol. 97, no. 1, pp. 42-47, 2006.

[31] J. Lehmann, M. Retz, C. Wiemers et al., "Adjuvant cisplatin plus methotrexate versus methotrexate, vinblastine, epirubicin, and cisplatin in locally advanced bladder cancer: results of a randomized, multicenter, phase III trial (AUO-AB 05/95)," Journal of Clinical Oncology, vol. 23, no. 22, pp. 4963-4974, 2005.

[32] "Adjuvant chemotherapy in invasive bladder cancer: a systematic review and meta-analysis of individual patient data Advanced Bladder Cancer (ABC) Meta-analysis Collaboration," European Urology, vol. 48, pp. 189-199, 2005.

[33] E. M. Ruggeri, D. Giannarelli, E. Bria et al., "Adjuvant chemotherapy in muscle-invasive bladder carcinoma: a pooled analysis from phase III studies," Cancer, vol. 106, no. 4, pp. 783-788, 2006.

[34] R. S. Svatek, S. F. Shariat, R. E. Lasky et al., "The effectiveness of off-protocol adjuvant chemotherapy for patients with urothelial carcinoma of the urinary bladder," Clinical Cancer Research, vol. 16, no. 17, pp. 44614467, 2010.

[35] F. Cognetti, E. M. Ruggeri, A. Felici et al., "Adjuvant chemotherapy (AC) with cisplatin + gemcitabine (CG) versus chemotherapy (CT) at relapse (CR) in patients (pts) with muscle-invasive bladder cancer (MIBC) submitted to radical cystectomy (RC). An Italian multicenter randomised phase III trial," in Proceedings of the American Society of Clinical Oncology (ASCO '08), vol. 5023, abstracts 26, 2008.

[36] W.M. Stadler, S. P. Lerner, S. Groshen et al., "Randomized trial of p53 targeted adjuvant therapy for patients (pts) with organ- confined node-negative urothelial bladder cancer (UBC)," in Proceedings of the American Society of Clinical Oncology (ASCO '09), vol. 5017, abstracts 27, 2009.

[37] L. G. Paz-Ares, E. Solsona, E. Esteban et al., "Randomized phase III trial comparing adjuvant paclitaxel/gemcitabine/cisplatin (PGC) to observation in patients with resected invasive bladder cancer: results of the Spanish Oncology Genitourinary Group (SOGUG) 99/01 study," in Proceedings of the American Society of Clinical Oncology (ASCO '10), vol. LBA4518, abstracts 28, 2010.

[38] C. N. Sternberg, "EORTC 30994 Summary," 2011.

[39] N. J. Rene, F. B. Cury, and L. Souhami, "Conservative treatment of invasive bladder cancer," Current Oncology, vol. 16, no. 4, pp. 36-47, 2009.

[40] C. Rödel, G. G. Grabenbauer, R. Kühn et al., "Combined-modality treatment and selective organ preservation in invasive bladder cancer: long-term results," Journal of Clinical Oncology, vol. 20, no. 14, pp. 3061-3071, 2002. 
[41] C. M. L. Coppin, M. K. Gospodarowicz, K. James et al., "Improved local control of invasive bladder cancer by concurrent cisplatin and preoperative or definitive radiation," Journal of Clinical Oncology, vol. 14, no. 11, pp. 2901-2907, 1996.

[42] N. D. James, S. A. Hussain, E. Hall et al., "Results of a phase III randomized trial of synchronous chemoradiotherapy (CRT) compared to radiotherapy (RT) alone in muscle-invasive bladder cancer (MIBC) (BC2001 CRUK/01/004)," in Proceedings of the American Society of Clinical Oncology (ASCO '10), vol. 4517, abstracts $28,2010$.

[43] W. U. Shipley, D. S. Kaufman, E. Zehr et al., "Selective bladder preservation by combined modality protocol treatment: long-term outcomes of 190 patients with invasive bladder cancer," Urology, vol. 60, no. 1, pp. 62-67, 2002.

[44] W. U. Shipley, K. A. Winter, D. S. Kaufman et al., "Phase III trial of neoadjuvant chemotherapy in patients with invasive bladder cancer treated with selective bladder preservation by combined radiation therapy and chemotherapy: initial results of Radiation Therapy Oncology Group 89-03," Journal of Clinical Oncology, vol. 16, no. 11, pp. 3576-3583, 1998.

[45] A. L. Zietman, W. U. Shipley, D. S. Kaufman et al., "A phase I/II trial of transurethral surgery combined with concurrent cisplatin, 5-fluorouracil and twice daily radiation followed by selective bladder preservation in operable patients with muscle invading bladder cancer," Journal of Urology, vol. 160, no. 5, pp. 1673-1677, 1998.

[46] C. Weiss, D. G. Engehausen, F. S. Krause et al., "Radiochemotherapy with cisplatin and 5-Fluorouracil after transurethral surgery in patients with bladder cancer," International Journal of Radiation Oncology Biology Physics, vol. 68, no. 4, pp. 1072-1080, 2007.

[47] D. S. Kaufman, K. A. Winter, W. U. Shipley et al., "The initial results in muscle-invading bladder cancer of RTOG 95-06: phase I/II trial of transurethral surgery plus radiation therapy with concurrent cisplatin and 5-fluorouracil followed by selective bladder preservation or cystectomy depending on the initial response," Oncologist, vol. 5, no. 6, pp. 471-476, 2000.

[48] D. S. Kaufman, K. A. Winter, W. U. Shipley et al., "Phase I-II RTOG study (99-06) of patients with muscleinvasive bladder cancer undergoing transurethral surgery, paclitaxel, cisplatin, and twice-daily radiotherapy followed by selective bladder preservation or radical cystectomy and adjuvant chemotherapy," Urology, vol. 73, no. 4, pp. 833-837, 2009.

[49] M. P. Hagan, K. A. Winter, D. S. Kaufman et al., "RTOG 97-06: initial report of a phase I-II trial of selective bladder conservation using TURBT, twice-daily accelerated irradiation sensitized with cisplatin, and adjuvant MCV combination chemotherapy," International Journal of Radiation Oncology Biology Physics, vol. 57, no. 3, pp. 665-672, 2003.

[50] E. Kent, H. Sandler, J. Montie et al., "Combined-modality therapy with gemcitabine and radiotherapy as a bladder preservation strategy: results of a phase I trial," Journal of Clinical Oncology, vol. 22, no. 13, pp. 2540-2545, 2004.

[51] V. K. Sangar, C. A. McBain, J. Lyons et al., "Phase I study of conformal radiotherapy with concurrent gemcitabine in locally advanced bladder cancer," International Journal of Radiation Oncology Biology Physics, vol. 61, no. 2, pp. 420-425, 2005.

[52] A. Choudhury, R. Swindell, J. P. Logue et al., "Phase II study of conformal hypofractionated radiotherapy with concurrent gemcitabine in muscle-invasive bladder cancer," Journal of Clinical Oncology, vol. 29, no. 6, pp. 733-738, 2011.

[53] P. J. Loehrer, L. H. Einhorn, P. J. Elson et al., "A randomized comparison of cisplatin alone or in combination with methotrexate, vinblastine, and doxorubicin in patients with metastatic urothelial carcinoma: a cooperative group study," Journal of Clinical Oncology, vol. 10, no. 7, pp. 1066-1073, 1992.

[54] C. J. Logothetis, F. H. Dexeus, L. Finn et al., "A prospective randomized trial comparing MVAC and CISCA chemotherapy for patients with metastatic urothelial tumors," Journal of Clinical Oncology, vol. 8, no. 6, pp. 1050-1055, 1990.

[55] C. N. Sternberg, P. H. M. De Mulder, J. H. Schornagel et al., "Randomized phase III trial of high-doseintensity methotrexate, vinblastine, doxorubicin, and cisplatin (MVAC) chemotherapy and recombinant human granulocyte colony-stimulating factor versus classic MVAC in advanced urothelial tract tumors: European 
organization for research and treatment of cancer protocol no. 30924," Journal of Clinical Oncology, vol. 19, no. 10, pp. 2638-2646, 2001.

[56] S. B. Saxman, K. J. Propert, L. H. Einhorn et al., "Long-term follow-up of a phase III intergroup study of cisplatin alone or in combination with methotrexate, vinblastine, and doxorubicin in patients with metastatic urothelial carcinoma: a cooperative group study," Journal of Clinical Oncology, vol. 15, no. 7, pp. 2564-2569, 1997.

[57] A. Bamias, G. Aravantinos, C. Deliveliotis et al., "Docetaxel and cisplatin with granulocyte colony-stimulating factor (G-CSF) versus MVAC with G-CSF in advanced urothelial carcinoma: a multicenter, randomized, phase III study from the Hellenic Cooperative Oncology Group," Journal of Clinical Oncology, vol. 22, no. 2, pp. 220-228, 2004.

[58] H. Von Der Maase, L. Andersen, L. Crinò, S. Weinknecht, and L. Dogliotti, "Weekly gemcitabine and cisplatin combination therapy in patients with transitional cell carcinoma of the urothelium: a phase II clinical trial," Annals of Oncology, vol. 10, no. 12, pp. 1461-1465, 1999.

[59] M. J. Moore, E. W. Winquist, N. Murray et al., "Gemcitabine plus cisplatin, an active regimen in advanced urothelial cancer: a phase II trial of the National Cancer Institute of Canada Clinical Trials Group," Journal of Clinical Oncology, vol. 17, no. 9, pp. 2876-2881, 1999.

[60] D. Kaufman, D. Raghavan, M. Carducci et al., "Phase II trial of gemcitabine plus cisplatin in patients with metastatic urothelial cancer," Journal of Clinical Oncology, vol. 18, no. 9, pp. 1921-1927, 2000.

[61] H. Von der Maase, S. W. Hansen, J. T. Roberts et al., "Gemcitabine and cisplatin versus methotrexate, vinblastine, doxorubicin, and cisplatin in advanced or metastatic bladder cancer: results of a large, randomized, multinational, multicenter, phase III study," Journal of Clinical Oncology, vol. 18, no. 17, pp. 3068-3077, 2000.

[62] H. Von Der Maase, L. Sengelov, J. T. Roberts et al., "Long-term survival results of a randomized trial comparing gemcitabine plus cisplatin, with methotrexate, vinblastine, doxorubicin, plus cisplatin in patients with bladder cancer," Journal of Clinical Oncology, vol. 23, no. 21, pp. 4602-4608, 2005.

[63] J. Bellmunt, V. Guillem, L. Paz-Ares et al., "Phase I-II study of paclitaxel, cisplatin, and gemcitabine in advanced transitional-cell carcinoma of the urothelium," Journal of Clinical Oncology, vol. 18, no. 18, pp. 3247-3255, 2000.

[64] J. Bellmunt, H. von der Maase, G. M. Mead et al., "Randomized phase III study comparing paclitaxel/cisplatin/gemcitabine (PCG) and gemcitabine/cisplatin (GC) in patients with locally advanced (LA) or metastatic (M) urothelial cancer without prior systemic therapy; EORTC30987/Intergroup Study," in Proceedings of the American Society of Clinical Oncology (ASCO '07), vol. LBA5030, abstracts 25, 2007.

[65] R. Petrioli, B. Frediani, A. Manganelli et al., "Comparison between a cisplatin-containing regimen and a carboplatin-containing regimen for recurrent or metastatic bladder cancer patients: a randomized phase II study," Cancer, vol. 77, no. 2, pp. 344-351, 1996.

[66] J. Bellmunt, A. Ribas, N. Eres et al., "Carboplatin-based versus cisplatin-based chemotherapy in the treatment of surgically incurable advanced bladder carcinoma," Cancer, vol. 80, no. 10, pp. 1966-1972, 1997.

[67] R. Dreicer, J. Manola, B. J. Roth et al., "Phase III trial of methotrexate, vinblastine, doxorubicin, and cisplatin versus carboplatin and paclitaxel in patients with advanced carcinoma of the urothelium: a trial of the Eastern Cooperative Oncology group," Cancer, vol. 100, no. 8, pp. 1639-1645, 2004.

[68] L. Dogliotti, G. Cartenì, S. Siena et al., "Gemcitabine plus cisplatin versus gemcitabine plus carboplatin as firstline chemotherapy in advanced transitional cell carcinoma of the urothelium: results of a randomized phase 2 trial," European Urology, vol. 52, no. 1, pp. 134-141, 2007.

[69] M. D. Galsky, A. Iasonos, S. Mironov, J. Scattergood, M. G. Boyle, and D. F. Bajorin, "Phase II trial of dosedense doxorubicin plus gemcitabine followed by paclitaxel plus carboplatin in patients with advanced urothelial carcinoma and impaired renal function," Cancer, vol. 109, no. 3, pp. 549-555, 2007.

[70] M. De Santis, J. Bellmunt, G. Mead et al., "Randomized phase II/III trial comparing gemcitabine/carboplatin (GC) and methotrexate/carboplatin/vinblastine (M-CAVI) in patients (pts) with advanced urothelial cancer (UC) unfit for cisplatin-based chemotherapy (CHT): phase III results of EORTC study 30986," in Proceedings of the American Society of Clinical Oncology (ASCO '10), vol. LBA4519, abstracts 28, 2010.

[71] H. Linardou, G. Aravantinos, E. Efstathiou et al., "Gemcitabine and carboplatin combination as first-line treatment in elderly patients and those unfit for cisplatin-based chemotherapy with advanced bladder carcinoma: phase II study of the Hellenic Co-operative Oncology Group," Urology, vol. 64, no. 3, pp. 479-484, 2004. 
[72] J. Bellmunt, R. De Wit, J. Albanell, and J. Baselga, "A feasibility study of carboplatin with fixed dose of gemcitabine in "unfit" patients with advanced bladder cancer," European Journal of Cancer, vol. 37, no. 17, pp. 2212-2215, 2001.

[73] D. J. Vaughn, J. Manola, R. Dreicer, W. See, R. Levitt, and G. Wilding, "Phase II study of paclitaxel plus carboplatin in patients with advanced carcinoma of the urothelium and renal dysfunction (E2896): a trial of the Eastern Cooperative Oncology group," Cancer, vol. 95, no. 5, pp. 1022-1027, 2002.

[74] M. D. Galsky, N. M. Hahn, J. E. Rosenberg et al., "Defining "cisplatin ineligible" patients with metastatic bladder cancer," in Proceedings of the American Society of Clinical Oncology (ASCO '11), vol. 238, abstracts 29, 2011.

[75] G. V. Raj, A. Iasonos, H. Herr, and S. M. Donat, "Formulas calculating creatinine clearance are inadequate for determining eligibility for cisplatin-based chemotherapy in bladder cancer," Journal of Clinical Oncology, vol. 24, no. 19, pp. 3095-3100, 2006.

[76] A. A. Meluch, F. A. Greco, H. A. Burris et al., "Paclitaxel and gemcitabine chemotherapy for advanced transitional-cell carcinoma of the urothelial tract: a phase II trial of the minnie pearl cancer research network," Journal of Clinical Oncology, vol. 19, no. 12, pp. 3018-3024, 2001.

[77] J. Li, B. Juliar, C. Yiannoutsos et al., "Weekly paclitaxel and gemcitabine in advanced transitional-cell carcinoma of the urothelium: a phase II Hoosier Oncology group study," Journal of Clinical Oncology, vol. 23, no. 6, pp. 1185-1191, 2005.

[78] C. N. Sternberg, F. Calabrò, G. Pizzocaro, L. Marini, S. Schnetzer, and A. Sella, "Chemotherapy with an every-2week regimen of gemcitabine and paclitaxel in patients with transitional cell carcinoma who have received prior cisplatin-based therapy," Cancer, vol. 92, no. 12, pp. 2993-2998, 2001.

[79] P. Albers, S.-I. Park, G. Niegisch et al., "Randomized phase III trial of 2nd line gemcitabine and paclitaxel chemotherapy in patients with advanced bladder cancer: short-term versus prolonged treatment [German Association of Urological Oncology (AUO) trial AB 20/99]," Annals of Oncology, vol. 22, no. 2, pp. 288-294, 2011.

[80] A. Vergnenégre, J. Tillon, R. Corre et al., "A randomized phase II trial assessing in advanced non-small cell lung cancer patients with stable disease after two courses of cisplatin-gemcitabine an early modification of chemotherapy doublet with paclitaxel-gemcitabine versus continuation of cisplatin-gemcitabine chemotherapy (GFPC 03-01 study)," Journal of Thoracic Oncology, vol. 4, no. 3, pp. 364-370, 2009.

[81] R. Dreicer, H. Li, M. M. Cooney, G. Wilding, and B. J. Roth, "Phase 2 trial of pemetrexed disodium and gemcitabine in advanced urothelial cancer (E4802): a trial of the Eastern Cooperative Oncology Group," Cancer, vol. 112, no. 12, pp. 2671-2675, 2008.

[82] K. Kanai, E. Kikuchi, T. Ohigashi et al., "Gemcitabine and paclitaxel chemotherapy for advanced urothelial carcinoma in patients who have received prior cisplatin-based chemotherapy," International Journal of Clinical Oncology, vol. 13, no. 6, pp. 510-514, 2008.

[83] T. Suyama, T. Ueda, S. Fukasawa et al., "Combination of gemcitabine and paclitaxel as second-line chemotherapy for advanced urothelial carcinoma," Japanese Journal of Clinical Oncology, vol. 39, no. 4, pp. 244-250, 2009.

[84] S. Krege, V. Rembrink, C. H. Börgermann, T. Otto, and H. Rubben, "Docetaxel and ifosfamide as second line treatment for patients with advanced or metastatic urothelial cancer after failure of platinum chemotherapy: a phase 2 study," Journal of Urology, vol. 165, no. 1, pp. 67-71, 2001.

[85] M. H. A. Hussain, G. R. MacVicar, D. P. Petrylak et al., "Trastuzumab, paclitaxel, carboplatin, and gemcitabine in advanced human epidermal growth factor receptor-2/neu-positive urothelial carcinoma: results of a multicenter phase II National Cancer Institute trial," Journal of Clinical Oncology, vol. 25, no. 16, pp. 2218-2224, 2007.

[86] D. E. Neal, L. Sharples, K. Smith, J. Fennelly, R. R. Hall, and A. L. Harris, "The epidermal growth factor receptor and the prognosis of bladder cancer," Cancer, vol. 65, no. 7, pp. 1619-1625, 1990.

[87] D. J. Gallagher, M. I. Milowsky, S. R. Gerst et al., "Phase II study of sunitinib in patients with metastatic urothelial cancer," Journal of Clinical Oncology, vol. 28, no. 8, pp. 1373-1379, 2010.

\section{This article should be cited as follows:}

Carrie Costantini and Frederick Millard, "Update on Chemotherapy in the Treatment of Urothelial Carcinoma," TheScientificWorldJOURNAL, vol. 11, pp. 1981-1994, 2011. 


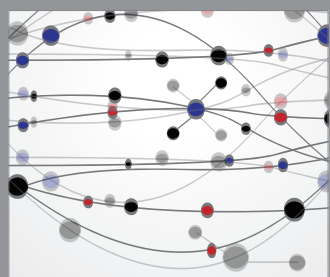

The Scientific World Journal
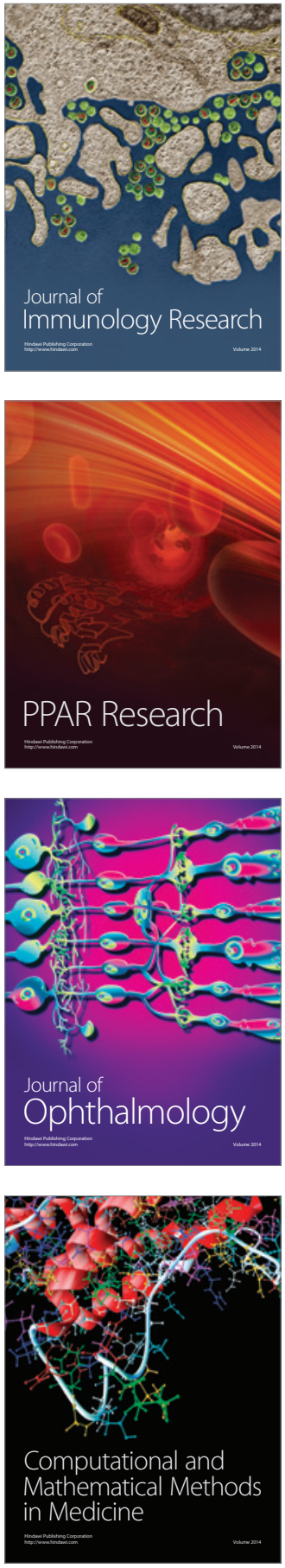

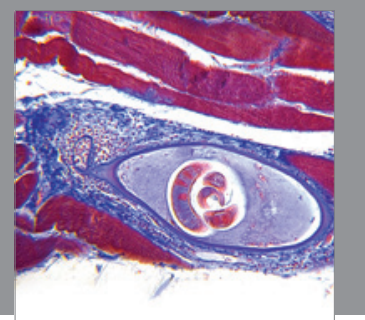

Gastroenterology

Research and Practice
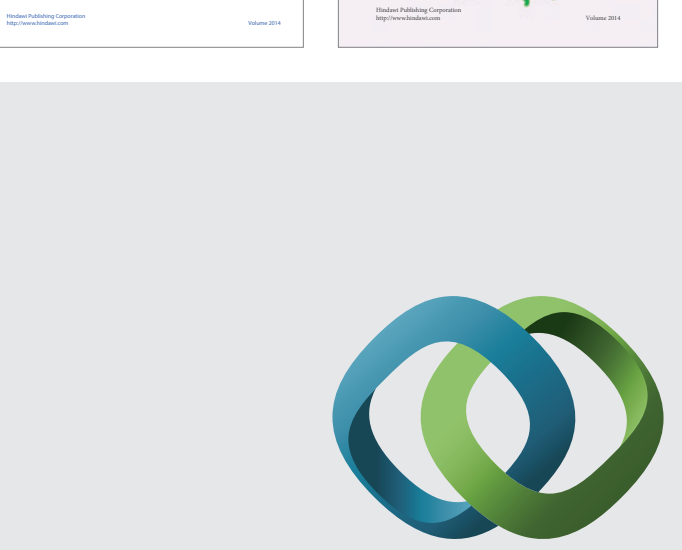

\section{Hindawi}

Submit your manuscripts at

http://www.hindawi.com
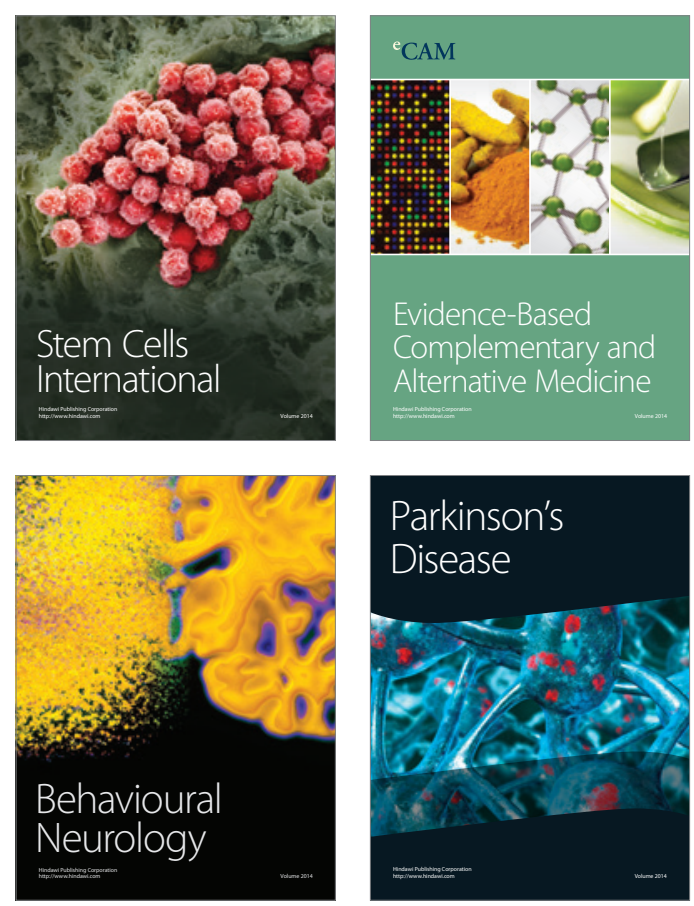

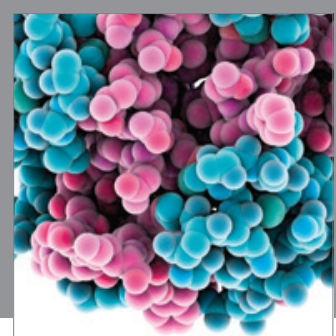

Journal of
Diabetes Research

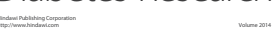

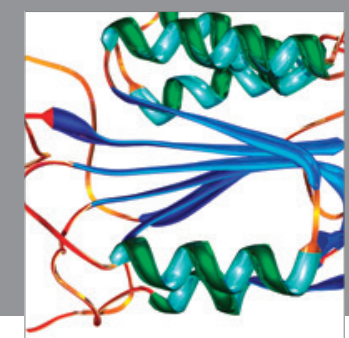

Disease Markers
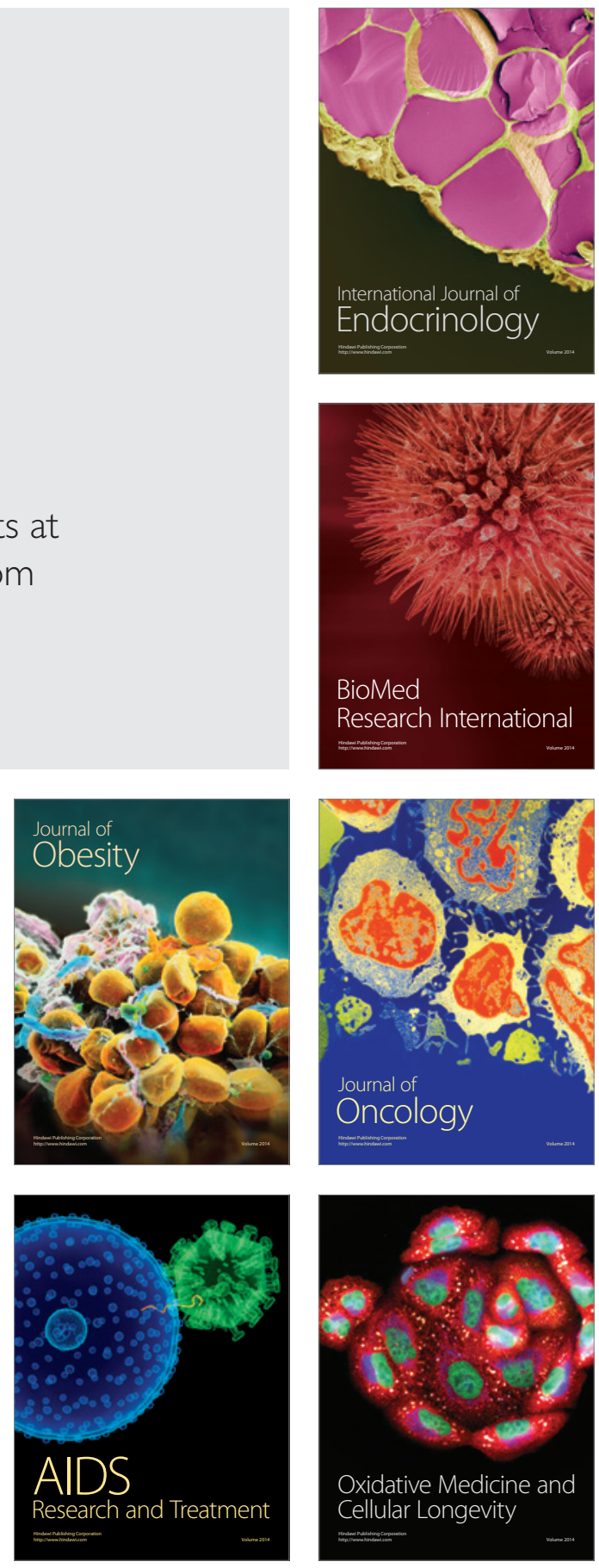